\title{
Leadership \& The Role of Information: Making The Creatively Informed Questioner
}

\section{By Stuart Basefsky, Published on October 29, 2008}

The quintessential leader is an informed leader. So it is said. However, this concept of being informed seems to lack definition and purpose. What does it mean to be informed? And what is the role of information in leadership?

People often think that leaders are informed because they are educated or quick learners. To some extent this may be true. A more realistic view would be that they use their education in a manner that positions themselves and those around them to succeed and sustain their success. How do they do this? An explanation follows.

Certainly good leadership is not dependent on academic degrees, certifications, and other types of qualification standards. These educational levels of distinction are helpful because they indicate that some potential ability has been acquired. All organizations want competent, educated, or at least bright people in leadership roles. Holding a leadership position does not mean that one is a good leader. Leadership authority does not come from an assigned, bureaucratic position in a hierarchy. Leadership authority can be enhanced by structure and position. But a true, authoritative leader is more than an assigned role as Prof. Bacharach, carefully explains in his many writings and workshops.

Common to the best leaders, however, is one distinguishing factor - the ability to use information creatively in raising questions. It is this ability to raise relevant, provocative, insightful, and often pathfinding questions that separates true leaders from those who may be occupying a leadership position. After all, there are all kinds of directors, managers, executives - people with titles. Few of us would say that their title makes them a leader. Several nouns are often used like judgment, persuasiveness, trust, and more to point to the many qualities necessary in a good leader. Easily forgotten, however, is the quality of being creatively informed.

Most students, workers, business executives and even academics make the mistake of thinking that having facts or fact-finding is a pre-requisite to good decision-making and authoritative leadership. This is misguided. Facts and fact-finding can support decisions, but most decisions are reached by being creatively informed enough to raise questions that lead individuals and groups to pursue new goals and ventures in an ever-changing world while shoring up and maintaining their successes. It also drives them to find more information or to create their own.

Having and gathering the information that reveals trends, patterns, tendencies, opportunities, weaknesses, strengths, and more is stuff of leadership when it is combined with creative efforts to raise the proper questions.

Information that is not coupled with a proclivity to question findings, direction, risk, mission and goals is relatively useless. For example, many business leaders ask their employees to benchmark other companies or find best practices. These are only useful when questioning how your own company might or might not benefit from the findings. Following benchmarks and best practices that are not a good fit 
for your company or its culture can be counterproductive. The most effective way of using benchmarks and best practices is as a creative mechanism for raising questions about your own company. Following what others do is rarely a form of good leadership.

Every good leader should be aware of the environment in which they operate and how other environments may alter their course. It is this awareness that makes them raise the proper questions. It makes them creatively informed. One needs to value the questions that information forces one to raise not the information itself. This being said, it is information properly consumed by the creative leader that can fire their intellect and abilities.

In upcoming commentaries, I will give concrete examples of how properly selected and disseminated information can improve leadership by keeping the fire of creative questioning lit.

Among the questions that will be addressed are:

- What is it that a good leader sees that others do not?

- How did they gain their insight?

- Where does one seek and find information that makes for a creatively informed leader?

Note: this article was first published on the Bacharach Blog: "This blog has been established for the purpose of observing and examining leadership. It is dedicated to the belief that good leaders do not simply come up with good ideas, but they have the capacity to actively put those ideas into play and deliver. This blog is dedicated to teaching, learning, and observing leadership in action, at all levels of our society-in the classroom, in the community, in the workplace, and in government. 